\title{
Phonological Development of Toddlers With Unilateral Cleft Lip and Palate Who Were Treated With and Without Infant Orthopedics: A Randomized Clinical Trial
}

\author{
Emmy M. Konst, M.A. \\ Toni Rietveld, Ph.D. \\ Herman F.M. Peters, Ph.D. \\ Birte Prahl-Andersen, D.D.S., Ph.D.
}

\begin{abstract}
Objective: To investigate the phonological development of toddlers from 2 to 3 years of age with complete unilateral cleft lip and palate (UCLP) treated during the first year of life with and without infant orthopedics (IO).

Design: In a randomized clinical trial (Dutchcleft), two groups of children were followed up: one treated with $\mathrm{IO}$ (IO group) and another that did not receive IO (non-IO group). Phonological skills were analyzed at 2, 2.5, and 3 years of age using a system for assessing phonological development of Dutch children (Fonologische Analyse van het Nederlands: FAN). The analysis included number of acquired consonants, order of phonological development, use of
\end{abstract} phonological processes, and occurrence of nasal escape.

Patients: Criteria for inclusion were complete UCLP, no soft tissue bands, no other malformations, parents fluent in Dutch, birth weight of a minimum of $2500 \mathrm{~g}$, and gestation time of a minimum of 38 weeks.

Interventions: 10 treatment based on a modified Zurich approach was started within 2 weeks after birth and used until soft palate closure at 12 months of age. Children in the non-IO group visited the clinic for an extra check-up at 6 weeks as well as before and after lip repair and soft palate closure. All other interventions were the same across groups.

Results: Phonological development of most 2.5-year-old IO children was normal or delayed. Most children in the non-IO group followed an abnormal developmental pattern. At age 3, the children in the 10 group had acquired more initial consonants. There were no group differences in the use of phonological processes or the occurrence of nasal escape.

Conclusions: Children treated with 10 during their first year of life followed a more normal path of phonological development between 2 and 3 years of age.

KEY WORDS: cleft lip and palate, infant orthopedics, phonological development, randomized prospective clinical trial

Several studies on child language development have described the continuity from babbling to early linguistic sound

\footnotetext{
Ms. Konst is Speech Language Pathologist, Department of Orthodontics and Oral Biology, and Dr. Rietveld is Associate Professor of Phonetics, Department of Language and Speech, University of Nijmegen, the Netherlands. Dr. Peters is Associate Professor of Speech Pathology, Department of Voice and Speech Pathology, University Medical Center, Nijmegen, the Netherlands. Dr. PrahlAndersen is Professor of Orthodontics, Department of Orthodontics, Academic Center for Dentistry, Amsterdam, the Netherlands

This research was supported by a grant from the National Health Insurance Board of the Netherlands. The research is part of the Dutch intercenter study into the effects of infant orthopedic treatment in complete UCLP (Dutchcleft), carried out in collaboration between the Cleft Palate Centers of the University of Nijmegen, Free University of Amsterdam, and Erasmus University of Rotterdam (coordinating orthodontists: A. M. Kuijpers-Jagtman and B. Prahl-Andersen).

Submitted December 2000; Accepted January 2002.

Address correspondence to: Emmy M. Konst, Department of Orthodontics and Oral Biology, University of Nijmegen, P.O. Box 9101, 6500 HB Nijmegen, the Netherlands. E-mail E.Konst@dent.kun.nl.
}

production (Oller et al., 1976; Stoel-Gammon, 1985; Vihman et al., 1985). Vihman et al. (1986) and Vihman and Miller (1988) analyzed the phonetic tendencies in the combined pool of word and babble vocalizations in English-speaking monolingual children without cleft over a 7-month period from the age of 9 to 16 months. Words were separated from nonwords in the phonetic analysis (consonant inventories). Only the first true consonant in a vocalization was analyzed. Large individual diversity was found in the use of consonant categories during the entire period. It was also found that at the point at which children used 15 words in a 30-minute session, considerably more babble than words was used in interaction. Words were typically short and included at least one consonant. Nonmeaningful vocalizations were predominantly lacking a true consonant, but by the 15 -word point, an increase in the use of consonants in babble vocalizations was observed. The overall increase in the use of babble vocalizations with true consonants reflected an increasing orientation toward language. 
Grunwell and Russell (1988) demonstrated that phonetic patterns that originated in prespeech may persist in later phonological development in children with cleft lip and palate. They described the phonological development of two children with complete cleft lip and palate from prespeech to the age of 3.5 years. Both children exhibited abnormal phonetic development in babble prior to palatal surgery but showed considerable individual variation in their later phonological development. In one of the subjects, persistent phonetic deviance seemed to restrict phonological development, but the other child developed a relatively normal phonological system once the palatal surgery provided an intact intraoral mechanism. The authors concluded that each of these subjects had his own route for phonetic and phonological development. A later study by Russell and Grunwell (1993) confirmed the considerable individual variation in phonological development of eight 2.5-year-old children with cleft (lip and) palate.

Other reports on phonological development in toddlers with cleft lip and palate have shown that these children often follow individual (and sometimes abnormal) patterns of phoneme acquisition. Lynch et al. (1983) demonstrated that children with cleft palate aged 29 to 37 months produced a disproportional number of words beginning with nasals and glides. Estrem and Broen (1989) also showed that 2-year-old children with cleft palate differed from a noncleft control group in that they tended to use fewer words beginning with [-sonorant] consonants and more words beginning with [-coronal] consonants. Chapman and Hardin (1992) compared the phonetic and phonological skills of 2-year-old children with cleft palate with children without cleft of the same age. Consonant inventories of the children's speech showed that both groups used a similar number of different consonant sounds. However, children with cleft palate were less accurate in their production of nasals and liquids than their normal peers. Two phonological processes, nasal assimilation and backing, were more frequently used in the cleft group. Frequent phonological processes in 2.5-year-old children with cleft lip and palate $(\mathrm{n}=8)$ investigated by Russell and Grunwell (1993) were palatalization, nasalization, glottalization, and backing of alveolars.

All studies cited above applied phonetic analysis (place and manner of articulation) or phonological process analysis to describe phonetic/phonological development. Process analysis provides more insight into the phonological abilities than articulation analysis, but it cannot give a complete assessment of the system of contrasts a child may have at his or her disposal. A more recent approach in the assessment of speech development is the use of nonlinear phonological theories. Both phonological process analysis and nonlinear frameworks use features of place, manner, and voicing in the classification of consonants, but they differ in that nonlinear phonology focuses on the hierarchical nature of relationships among these features (Bernhardt and Stoel-Gammon, 1994). A hierarchical representation reduces the number and types of phonological rules necessary to describe the sound patterns of a language. The hierarchical relationship between features is represented in a feature geometry (Fig. 1). In a nonlinear approach, the

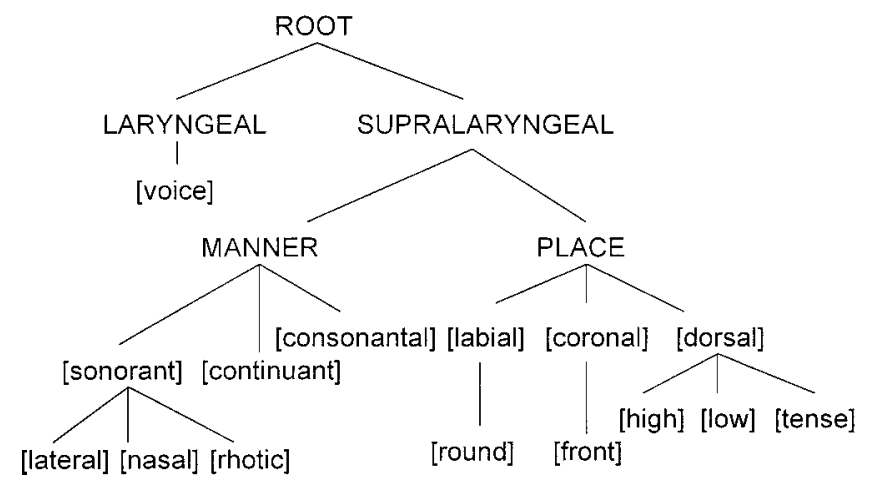

FIGURE 1 The feature hierarchy used in the Fonologische Analyse van het Nederlands (FAN) analysis (Beers, 1995).

primary focus is on the child's productions. When the child's form is identical to the adult's form, it is said to match; a nonmatch appears when there are differences between the two forms. From this point of view, nonmatches are a consequence of a developing system that differs from the system used by adult speakers. During the period of phonological acquisition, the child gradually adds specified features to the set of universally unspecified features (the default features) and in this way reorganizes its system of phonological contrasts.

In this study, a system for the assessment of phonological development of Dutch children that is based on nonlinear phonology (Beers, 1995) was used for evaluating the phonological development of children with a unilateral complete cleft lip and palate (UCLP) in relation to treatment with infant orthopedics (IO). Children born with a cleft lip and palate are often treated with IO. This treatment intends to guide the growth and position of the maxillary segments, to improve feeding and tongue posture, and is furthermore believed to aid speech and language development. Opinions about the value of IO treatment differ throughout the world (Winters and Hurwitz, 1995). Proponents state that this treatment facilitates speech development by creating a better oral structure to practice sounds and articulation movements (Stuffins, 1981; Hotz et al., 1986; Gnoinski, 1990; Gruber, 1990). As the plate obturates the cleft, it may facilitate the production of consonantlike elements (contoids) with high intraoral pressure build-up and the use of alveolar articulations. Opponents, however, believe that a plate reduces the tactile and kinesthetic proprioception of the tongue (Dorf et al., 1985) and that speech is negatively influenced by delayed hard palate closure, which is inherent to this treatment (Witzel et al., 1984; Winters and Hurwitz, 1995).

A type of IO used for treating children with UCLP is the Zurich approach, described by Hotz $(1979,1983)$. A prospective clinical trial into the effects of IO treatment based on a modified Zurich approach was started in 1993 (Kuijpers-Jagtman et al., 1998; Severens et al., 1998; Konst et al., 1999, 2000). Two groups are being followed up in the trial: a group of children treated with IO and a group who did not receive this therapy. In the IO group, the plate was worn day and night until soft palate closure at the age of 12 months. Speech and language development is one of the aspects that are investi- 
gated in this trial. Assessment of prelexical development of the two groups at age 12 months (just prior to soft palate surgery) and at age 18 months showed that IO facilitated the production of alveolar contoids in the 12-month-olds (Konst et al., 1999). All other characteristics of consonant production were the same in both groups. In the 18-month-old children, there was no longer a difference between the two groups regarding consonant production. It should be noted that the appliance was worn until the age of 12 months, at which time soft palate surgery was performed. It was concluded that IO influenced contoid production during the period of application, but the effect seemed to disappear after removal of the plate. Definitive conclusions could not be drawn. It is hypothesized, however, that because phonetic preferences originated in babbling may persist in later linguistic development, treatment with IO may result in less deviant phonological development. The purpose of the present study was to evaluate effects of IO treatment on phonological development of toddlers from 2 to 3 years of age. To this end, the two groups in our trial (IO group and non-IO group) were followed up at 6-month intervals until the age of 3 years.

\section{METHODS}

\section{Subjects}

The subjects in this study have all participated in a Dutch intercenter prospective clinical trial (called Dutchcleft) on the effects of IO. Babies with complete UCLP without soft tissue bands were recruited within 2 weeks after birth and randomly assigned to one of two groups by means of computerized balancing with regard to alveolar cleft width and birth weight. One group received IO according to a modified Zurich approach (Hotz, 1979, 1983), but the other group did not receive this treatment. All other interventions were the same in both groups. Primary lip repair (Millard technique) was performed at 18 weeks. The palate was closed in two stages, with soft palate closure at 12 months using the modified Von Langenbeck procedure. Hard palate surgery is usually delayed until approximately 9 years of age. The appliance was worn 24 hours a day until soft palate closure. However, two subjects stopped wearing the plate at an earlier stage because they rejected the appliance. All babies were Caucasian and their parents were fluent in Dutch. None of the babies had been diagnosed as having cognitive or neurological impairment or other congenital malformations.

The children were followed up at 6-month intervals from 2 ( 2 years, 0 months) to 3 years. In the 2.0 years age group, 16 children were assessed ( $9 \mathrm{IO}$ and 7 non-IO). At the age of 2.6 years, 18 children were evaluated (9 IO and 9 non-IO). At 3 years of age, 12 children (6 IO and 6 non-IO) participated in the speech recordings. The patients in the groups differed at the various time points: only six children (four IO and two non-IO) were included in the phonological analysis at all three ages.

\section{Data Collection}

All recordings were made in the child's home environment using high-quality audio equipment (TCD-D7 DAT Walkman with a Sennheiser MD421U-4 dynamic microphone; Sony, Tokyo, Japan) and an NV-M40E video camera (Panasonic, Osa$\mathrm{ka}$, Japan) while the child was engaged in free play with one of the researchers. A standardized set of age-appropriate toys was used to elicit speech. The toys were selected to represent words that are seen in the expressive vocabulary of the normally developing 2-year-old child (Schlichting et al., 1995). Most words contained singleton consonants in a one- or twosyllable structure. We ensured that each recorded conversational sample contained a minimum of 100 well-recorded utterances and that it was representative of the child's speech according to the parent.

\section{FAN System for Phonological Assessment}

The phonological development of the subjects was evaluated using a system for the assessment of phonological development of Dutch children based on nonlinear phonology (Beers, 1995). This system, referred to as Fonologische Analyse van het Nederlands (FAN), makes use of a feature hierarchy based on proposals that have been widely accepted by phonologists. The model represents hierarchical relations between higherlevel and lower-level features separately for features under a manner node and place node. Furthermore, the order in which children usually acquire the system of contrastive features is accounted for in the model. Segments with higher-level features are acquired before segments that have a more complex (lower-level) feature representation. In accordance with the constructionist view, the model allows for individual variation in the order of acquisition. However, this variation is restricted by the dominance relations between features in the feature geometry. The feature hierarchy used in the FAN analysis is shown in Figure 1. In this hierarchy, the supralaryngeal node dominates the manner node and place node. The class nodes under the place node correspond to the three basic places of articulation for Dutch: labial (/p, b, f, v, m/), coronal (/t, d, s, $\left.\mathrm{z}, \int, 3, \mathrm{n}, \mathrm{j} /\right)$, and dorsal (/k, x, $\mathrm{y} /$ ). The manner node dominates the features [consonantal], [sonorant], and [continuant]. Early in the development, the child has a reduced version of the feature hierarchy at his or her disposal, consisting only of the laryngeal node and the supralaryngeal place and manner node. The first expansion of this system is the appearance of the feature [consonantal]. Later on segments specified by the features [sonorant] or [continuant] are acquired. This order of acquisition is related to the concept of markedness in the sense that unmarked elements are acquired before marked elements.

To classify phonological development, Beers (1995) formulated five degree-of-complexity subgroups (Table 1). The degrees reflect expansions of the feature hierarchy in normal development based on the acquisition of initial consonants. Other longitudinal studies addressing the acquisition of Dutch largely found the same order of acquisition (Fikkert, 1998). 
TABLE 1 The Degree-of-Complexity Subgroups Formulated by Beers (1995)

\begin{tabular}{clc}
\hline Degree & \multicolumn{1}{c}{ Acquired Contrasts } & Acquisition at Age \\
\hline 1 & [sonorant], [labial], [coronal] & $1 ; 3-1 ; 8 \mathrm{y}$ \\
2 & [dorsal] & $1 ; 9-1 ; 11 \mathrm{y}$ \\
3 & [continuant] & $2 ; 0-2 ; 2 \mathrm{y}$ \\
4 & [front], [round] & $2 ; 3-2 ; 5 \mathrm{y}$ \\
5 & [lateral], [rhotic], [nasal] & $2 ; 6-2 ; 8 \mathrm{y}$ \\
\hline
\end{tabular}

Fikkert, however, provided evidence for a more flexible order in the acquisition of manner of articulation features. Her results showed that the class of plosives is acquired first, followed by the class of nasals, but children may vary in the order in which they acquire fricatives, liquids, and glides.

A contrastive system of the first degree according to Beers (1995) is restricted to the features [sonorant], [labial], and [coronal]. In normal development, [sonorant] is acquired by the contrast between $/ \mathrm{p} /$ and $/ \mathrm{m} /$ or between $/ \mathrm{t} /$ and $/ \mathrm{n} /$. The feature [consonantal] occurs as the first feature in normal phonological development. It is therefore considered to be the default feature in the contrastive system and is not incorporated in the degree of complexity. Children with cleft palate often have difficulties in mastering segments that account for the default feature [consonantal], such as stop consonants that require high intraoral pressure. They usually have fewer problems with segments specified by the feature [sonorant]. If stop consonants are absent in the consonant inventory, the child's contrastive system will not even achieve the first degree of complexity because the contrast [sonorant]-[consonantal] is not acquired. The child's development is then automatically classified as abnormal. Jansonius-Schultheiss (1999) also recognized this difficulty and suggested including the feature [sonorant] as the default feature when assessing the phonological development of children with cleft palate. The feature [sonorant] characterizes both consonantal segments (nasals, /l/ and /r/) and nonconsonantal segments (glides). By including this new default feature, the linguistic knowledge and phonological development of the child with cleft palate may be accounted for in a better way. Jansonius-Schultheiss (1999) also suggested allowing more patterns of individual variation. She allowed the acquisition of the lower-level-place features [round] and [front] before the acquisition of the higher-level-place features [labial] and [coronal]. Beers (1995) classified this type of acquisition as abnormal because the hierarchical order of acquisition is violated. We agree with Beers that if the hierarchical order is violated, the pattern of acquisition should be classified as abnormal.

The FAN analysis is based on 100 words obtained from a sample of spontaneous speech. In case a speech sample does not contain 100 true words, phonological analysis may also be performed on the basis of smaller samples (Beers, 1995). Although some reports indicate that results do not differ for words extracted from spontaneous speech and words elicited by means of an articulation test (Chapman, 1993), a sample of the child's spontaneous speech is preferred because it provides the researcher with a more representative picture of the child's linguistic abilities in everyday situations than a structured test situation does (Grunwell, 1993; Beers, 1995). Imitations by the child are not excluded from the analysis because imitations that occur in spontaneous speech are voluntary and reflect the child's phonological abilities.

\section{Procedure}

Sixteen children (9 IO and 7 non-IO) were analyzed at age 2 years. Because of the limited lexical development of these 2-year-olds, phonological analysis was performed on a sample of 50 words. Phonological analysis at age 2.5 years was based on a sample of 100 words. Eighteen children of this age (9 IO and 9 non-IO) were included in the analysis. At the age of 3 years, 12 children (6 IO and 6 non-IO) were included in the phonological analysis, which was also based on a sample of 100 words.

The first step in the analysis according to FAN was the phonetic transcription. Narrow phonetic transcriptions were made by a trained listener who was blinded as to the treatment the subjects received. Six randomly chosen recordings were also transcribed by a second trained listener to assess listener agreement. For the transcription of consonants, agreement between the listeners was calculated by dividing the number of agreements by the number of agreements plus disagreements. The agreement yielded $82 \%$ for all consonants regardless of word position.

Once transcribed, the sounds that were acquired by the child were surveyed. As suggested by Beers (1995), only syllable initial consonants were included in the inventory of acquired consonants. We also followed Beers (1995) in our definition of consonant acquisition. A consonant was considered acquired by the child if the consonant was attempted at least twice and the percentage of correct production was at least $75 \%$. Second, the order of phonological development (normal, delayed, or abnormal) was analyzed. The developmental order was determined by the system of contrasts that is acquired by the child. The child's contrastive system was compared with what is expected for his or her age by using the degrees of complexity (Table 1). The development is normal if the child's contrastive system corresponds to what can be expected for his or her age. A delayed phonological development occurs when the child uses a contrastive system that corresponds to a younger age group. Abnormal phonological development is characterized by violation of the hierarchical order of contrasts. For example, if a child has acquired the lower-levelplace features [round] and [front] before the higher-level-place features [labial] and [coronal], the child has acquired lowerlevel contrasts before higher-level contrasts under the same node. Finally, the occurrence of cleft-related phonological processes such as backing, nasalization, and glottalization as well as the occurrence of nasal escape were investigated. All consonants (regardless of syllable position) were included in this part of the FAN analysis. 
TABLE 2 Mean Number and Standard Deviation of Number of Acquired Consonants per Group, at Age 2, 2.5, and $3 \mathrm{y}^{* \dagger}$

\begin{tabular}{lrcc}
\hline Age & IO Group & Non-IO Group & Significance \\
\hline $2 \mathrm{y}$ & $3.9(\mathrm{n}=9)$ SD 3.8 & $1.4(\mathrm{n}=7)$ SD 1.1 & $t_{14}=1.66 ; p=.12$ \\
$2.5 \mathrm{y}$ & $8.0(\mathrm{n}=9)$ SD 3.7 & $4.1(\mathrm{n}=9)$ SD 2.1 & $t_{16}=2.70 ; p=.02$ \\
$3 \mathrm{y}$ & $12.2(\mathrm{n}=6)$ SD 2.8 & $5.7(\mathrm{n}=6)$ SD 2.7 & $t_{10}=4.08 ; p=.00$ \\
\hline
\end{tabular}

* The significance level after Bonferroni correction was $\alpha=0.016$.

$\dagger \mathrm{IO}=$ infant orthopedics; SD = standard deviation

\section{Statistics}

It was hypothesized that IO treatment would influence phonological development of the children with UCLP. More specifically, it was supposed that the IO group would acquire more initial consonants and exhibit a more advanced system of phonological contrasts than the non-IO group. Student's $t$ tests were used to evaluate differences in the number of acquired consonants and percent occurrence of phonological processes and nasal escape. All percentages were transformed into arcsin values before statistical tests were performed. To test the difference in the order of phonological development between the two treatment groups, an exact chi-square test was used. An analysis of variance (ANOVA) with repeated measures and concomitant simple main effect analysis was applied to evaluate differences for six subjects who were included at all three time points. We are aware of the fact that chance capitalization could occur by applying series of Student's $t$ and chi-square tests; therefore, the significance level was adjusted by means of a Bonferroni correction to $\alpha=0.016$ for the variables "acquired consonants" and "order of phonological development" and to $\alpha=0.004$ for the variable "phonological processes."

\section{RESULTS}

\section{Acquired Consonants}

First, the effects of treatment with IO on the number of initial consonants that had been acquired by the children was assessed (Table 2). At the age of 2 years, the mean number of acquired consonants in the IO group was 3.9, compared with a mean of 1.4 acquired consonants in the non-IO group. A Student's $t$ test showed that at this age, this difference was not significant $\left(t_{14}=1.66 ; p=.12\right)$. At age 2.5 years, the children in the IO group had acquired a higher number of consonants (8.0) than the children in the non-IO group (4.1). However, the difference was not statistically significant after Bonferroni correction $\left(t_{16}=2.70 ; p=.02\right)$. At age 3 years, the mean number of acquired consonants was significantly higher in the group that had been treated with IO $\left(t_{10}=4.08 ; p=.002\right)$, even after Bonferroni correction. The children in the IO group had a mean number of 12.2 acquired consonants, compared with a mean of 5.7 acquired consonants in the non-IO group.

\section{Order of Phonological Development}

After assessment of the acquired consonants, the effect of IO on the order of phonological development was analyzed. Three types of development were distinguished: normal development, delayed development, and abnormal development. As mentioned in the procedure, development is determined by the contrastive system used by the child. If the child's contrastive system corresponded to what can be expected for his or her age (in terms of degrees of complexity) the phonological development was considered normal. A delayed phonological development occurred when the child used a contrastive system that corresponds to a younger age group. Abnormal phonological development was characterized by violation of the hierarchical order of contrasts (Table 3). At the age of 2 years, phonological development of two children in the IO group was classified as normal and two were classified as delayed. Five children in this group followed an abnormal pattern of acquisition. All seven children in the non-IO group developed an abnormal system of phonological contrasts. A chi-square test was used to determine whether there was a statistically significant difference between the groups in the order of phonological development. This was not the case (chi-square ${ }_{2}=3.39$; $p=.09)$.

At 2.5 years of age, the system of phonological contrasts that had been acquired by the children was closer to normal in the IO group, compared with the non-IO group. The development of only one child in the IO group was characterized as abnormal, whereas eight children in the non-IO group had developed an abnormal system of phonological contrasts. A chi-square test showed that the groups differed significantly in the acquisition of phonological contrasts (chi-square $_{2}=9.84$; $p=.002)$. At the age of 3 years, all children in the IO treatment group had developed a normal $(n=4)$ or delayed $(n=$ 2) system of contrasts; none of these children followed an abnormal pattern of acquisition. In the non-IO group, the phonological development of two children was classified as abnormal because the system of contrasts that they used violated the hierarchical order of acquisition of contrasts. The other

TABLE 3 Order of Phonological Development of Children at Age 2, 2.5, and $3 \mathrm{y}^{* \dagger}$

\begin{tabular}{|c|c|c|c|c|c|c|c|c|c|}
\hline & \multicolumn{3}{|c|}{ Age $2 y$} & \multicolumn{3}{|c|}{ Age $2.5 y$} & \multicolumn{3}{|c|}{ Age $3 y$} \\
\hline & Normal & Delayed & Abnormal & Normal & Delayed & Abnormal & Normal & Delayed & Abnormal \\
\hline IO & $\mathrm{n}=2$ & $\mathrm{n}=2$ & $\mathrm{n}=5$ & $\mathrm{n}=4$ & $\mathrm{n}=4$ & $\mathrm{n}=1$ & $\mathrm{n}=4$ & $\mathrm{n}=2$ & $\mathrm{n}=0$ \\
\hline Non-IO & $\mathrm{n}=0$ & $\mathrm{n}=0$ & $\mathrm{n}=7$ & $\mathrm{n}=0$ & $\mathrm{n}=1$ & $\mathrm{n}=8$ & $\mathrm{n}=0$ & $\mathrm{n}=4$ & $\mathrm{n}=2$ \\
\hline Sign & \multicolumn{3}{|c|}{$\chi^{2}{ }_{2}=3.39 p=.09$} & \multicolumn{3}{|c|}{$\chi^{2}{ }_{2}=9.84 p=.002$} & \multicolumn{3}{|c|}{$\chi_{2}^{2}=5.82 p=.03$} \\
\hline
\end{tabular}

* The significance level after Bonferroni correction was $\alpha=0.016$.

$\dagger \mathrm{IO}=$ infant orthopedics. 
TABLE 4 Mean Frequency of Occurrence and Standard Deviation of Phonological Processes and Nasal Escape (in Percentages) at Age $2 * \dagger$

\begin{tabular}{lccc}
\hline & $I O(n=9)$ & Non-IO $(n=7)$ & Sign \\
\hline Backing, \% & 7.7, SD 7.7 & 20.5, SD 15.2 & $t_{14}=-1.73 ; p=.11$ \\
Nasalization, \% & 9.7, SD 9.3 & 5.5, SD 4.2 & $t_{14}=0.83 ; p=.42$ \\
Glottalization, \% & 4.4, SD 5.4 & 7.4, SD 14.1 & $t_{14}=-0.36 ; p=.73$ \\
Nasal escape, \% & 4.0, SD 2.6 & 12.3, SD 7.1 & $t_{14}=-3.28 ; p=.005$ \\
\hline
\end{tabular}

* The significance level after Bonferroni correction was $\alpha=0.004$.

$\dagger \mathrm{IO}=$ infant orthopedics; $\mathrm{SD}=$ standard deviation.

children $(n=4)$ in the non-IO group were delayed in their phonological development. At this age, the difference in order of phonological development between the two groups was not statistically significant (chi-square ${ }_{2}=5.82 ; p=.03$ ).

\section{Phonological Processes and Nasal Escape}

The third variable that was investigated was phonological process usage and occurrence of nasal escape. The occurrence of these cleft-related processes was expressed as the percentage occurrence for the total number of consonants targeted by the child. To test differences in the occurrence of these processes, the percentages were transformed into arcsin values before Student's $t$ tests were performed. Statistical analysis (Table 4) showed that in the 2-year-old group, only the difference between the groups in occurrence of nasal escape approached statistical significance. In the non-IO group, more nasal escape was present during consonant articulation, compared with the IO group $\left(t_{14}=-3.28 ; p=.005\right)$; however, after Bonferroni correction this difference was not statistically significant. IO treatment did not affect any of the phonological processes that were investigated in the 2.5 -year-old children (Table 5). Nasal escape occurred more often in the speech of children who had not been treated with IO, but statistical significance was not reached $\left(t_{16}=-2.07 ; p=.06\right)$. The backing process is the only cleft-related phonological process that was present in more than $10 \%$ of the consonant articulations. Here, too, occurrence in the non-IO group was not statistically different from occurrence in the IO group $\left(t_{16}=-1.41 ; p=.18\right)$. Analysis of the cleft-related processes at age 3 (Table 6) showed that there were no significant differences between the two groups. All phonological processes and nasal escape occurred at the same extent in both groups at this age.

\section{Longitudinal Analysis}

In the analysis described in the sections above, the subjects differed at the various time points. A longitudinal analysis could be performed for only six children (four IO and two non-IO) from the cohort in this study who were included in all three time points. The data from this group were tested by means of an ANOVA with repeated measures to evaluate differences over time for the same group of patients. For the acquired number of consonants, there was a difference between the groups at age $2.5\left(\mathrm{~F}_{1,4}=21.01 ; p=.01\right)$ and also
TABLE 5 Mean Frequency of Occurrence and Standard Deviation of Phonological Processes and Nasal Escape (in Percentages) at Age $2.5^{*} \dagger$

\begin{tabular}{lccl}
\hline & $I O(n=9)$ & Non-IO $(n=9)$ & Sign \\
\hline Backing, \% & 10.4, SD 10.2 & 21.1, SD 14.2 & $t_{16}=-1.41 ; p=.18$ \\
Nasalization, \% & 5.2, SD 6.2 & 2.3, SD 2.3 & $t_{16}=1.02 ; p=.32$ \\
Glottalization, \% & 2.0, SD 4.6 & 2.1, SD 4.1 & $t_{16}=-0.35 ; p=.72$ \\
Nasal escape, \% & 5.7, SD 4.0 & 10.0, SD 5.2 & $t_{16}=-2.07 ; p=.06$ \\
\hline
\end{tabular}

* The significance level after Bonferroni correction was $\alpha=0.004$.

$\dagger \mathrm{IO}=$ infant orthopedics; SD = standard deviation.

at age $3\left(\mathrm{~F}_{1,4}=57.9 ; p=.002\right)$. At both time points, the IO children had acquired more initial consonants than the non-IO children. The results at age 3 years are in accordance with the analysis in which the subjects differ at the three time points. However, contrary to the analysis described in the previous section, the analysis with repeated measures also showed a significant difference in acquired consonants at age 2.5 years.

For the phonological process usage and the occurrence of nasal escape, the results were similar to the analysis described in the previous section. There was one exception: in the longitudinal analysis, the groups differed in the occurrence of nasal escape at the age of 3 years. In the non-IO children's speech, statistically significant more nasal escape occurred $\left(\mathrm{F}_{1,4}\right.$ $=45.4 ; p=.003$ ).

\section{Discussion}

This article describes the effects of IO treatment on the phonological development of children from age 2 to 3 years. A previous report (Konst et al., 1999) involving the same group of patients at a younger age ( 1 and 1.5 years) showed that treatment with IO facilitates the production of alveolar contoids in the prelexical period. This effect was present only during the period in which IO was used. At the age of 1.5, when the soft palate was closed and the plate was no longer used, the effect seemed to disappear.

The results of phonological analysis according to the FAN method described in this article show that IO treatment also affected the speech development of the children at an older age. The order in which children acquired contrastive features was influenced by treatment with IO. In the IO group, fewer children followed an abnormal developmental pattern than the non-IO children. This difference was statistically significant at 2.5 years of age. These results suggest that the children who

TABLE 6 Mean Frequency of Occurrence and Standard Deviation of Phonological Processes and Nasal Escape (in Percentages) at Age $3 * \dagger$

\begin{tabular}{lccc}
\hline & $I O(n=6)$ & Non-IO $(n=6)$ & Sign \\
\hline Backing, \% & 5.5, SD 5.9 & 14.5, SD 14.5 & $t_{10}=-1.01 ; p=.34$ \\
Nasalization, \% & 0.9, SD 0.1 & 3.1, SD 2.6 & $t_{10}=-1.66 ; p=.13$ \\
Glottalization, \% & 0.0, SD 0.0 & 1.5, SD 1.7 & $t_{10}=-2.64 ; p=.03$ \\
Nasal escape, \% & 4.1, SD 1.8 & 8.7, SD 4.8 & $t_{10}=-1.75 ; p=.11$ \\
\hline
\end{tabular}

$*$ The significance level after Bonferroni correction was $\alpha=0.004$.

$\dagger \mathrm{IO}=$ infant orthopedics; SD = standard deviation. 
were treated with IO seemed to have less difficulty in acquiring a normal phonological system than the children who did not have IO treatment. An explanation for this finding could be that IO in the first year of life provides the child with a better oral structure to practice sounds and articulation movements because the cleft in the alveolus and the palate is covered with the appliance. The tongue finds a better support for the production of alveolar sounds, which results in a higher use of these sounds in babbling at 1 year of age. The establishment of more normal speech motor patterns in babbling may result in a more normal phonological development at a later age because it is generally accepted that patterns in babbling correspond to patterns in first words. The influence of the child's speech production abilities on development of the early vocabulary of children with cleft palate has been pointed out by Estrem and Broen (1989). They found that the lexicons of 2year-old children with cleft palate included more words beginning with [+sonorant] phonemes than the lexicons of children without cleft. Furthermore, the lexicons of children without cleft contained more words with initial lingua-alveolar phonemes, whereas children with cleft palate tended to target more words with labial and glottal sounds.

Another difference that was observed in relation to treatment with IO in this study was the number of acquired (initial) consonants. At the age of 3 , the children who were treated with the IO had acquired more (initial) consonants than the non-IO children. This effect was also present in the younger children, but these differences approached only statistical significance. It is not coincidental that the group who developed the system of phonological contrasts in a normal or delayed manner had acquired more consonants than the group with abnormal developmental patterns. The acquisition of new segments through expansion of the system of phonological contrast may progress more efficiently in a normal developmental pattern.

As the children grew older, there was an increase in the number of acquired consonants and a decrease in the number of children who developed an abnormal phonological system of contrasts. At age 3, all IO children used a system of phonological contrasts that was age appropriate or delayed. In the non-IO group, there was also a decrease in the number of children who used an abnormal system of contrast, but this seemed to be at a later age than in the IO group. These results suggest that treatment with IO enhanced the phonological development in the period from 2 to 3 years. However, it can be expected that both groups will catch up in phonological development. The literature shows that the period from 2 to 4 years in normal children is marked by a rapid development in speech sound acquisition (Grunwell, 1982). At the age of 4, most phonological processes have disappeared in normally developing children. Chapman (1993) concluded that at the age of 5 , the phonological process usage of children with cleft palate is similar to their noncleft peers, but at an earlier age (3 and 4 years) children with cleft palate more frequently employ common phonological processes in their speech. Chapman (1993) argues that these findings support the view that children's early phonological simplifications occur as a result of articulatory incompetence.

The use of phonological processes and the occurrence of nasal escape were also investigated in this study. There were no statistically significant differences in process usage between the two groups. Additionally, it should be noted that the percent occurrence of these processes in our study was small, which is in accordance with the literature (Chapman and Hardin, 1992). Thus, it is unlikely that if a difference in occurrence of these processes related to IO would have occurred, it would be considered clinically significant.

It is important to mention the fact that only six children (four IO and two non-IO) in this study were evaluated at all three time points. Although the study was longitudinal, the subjects in the groups differed at the three time points. This was because of the fact that 14 of the 2-year-old subjects included in the trial failed to produce enough lexical utterances for the FAN analysis. The number of ineligible subjects was almost equal in both treatment groups (six IO and eight nonIO), so their exclusion at 2 years of age did not appear to affect the outcome of the study. However, the fact that only temporal effects were found (i.e., an effect in one age group but not in the others) was probably related to the differing subjects in the three age groups. An ANOVA with repeated measures was carried out on the six children who were included in the analysis at all time points to evaluate differences over time for the same group of patients. This analysis revealed a more longitudinal effect of IO on the number of acquired consonants (i.e., at age 2.5 and 3 years) than the separate analyses of groups at the three different time points (i.e., an effect only at 3 years). Furthermore, an effect in the occurrence of nasal escape at age 3 occurred that was not present in the separate analysis at this time point. All other results were the same. The fact that for these two very small groups (IO $\mathrm{n}$ $=4$, non-IO $\mathrm{n}=2$ ) effects were found in the analysis with repeated measures suggests that IO treatment affected the phonological development of the children in this trial.

\section{CONCLUSIONS}

Children with complete UCLP who were treated with IO based on a modified Zurich approach in their first year of life followed a more normal order of phonological development between 2 and 3 years of age. Compared with children who did not undergo IO treatment, the IO treatment group had acquired more (initial) consonants at age 3. At 2.5 years of age, the system of phonological contrasts was normal or delayed in most IO children, but most non-IO children followed an abnormal developmental pattern. An earlier report on the Dutchcleft study showed that at 12 months of age, IO facilitates the production of alveolar consonants. The better opportunity in the IO group to practice these sounds in babbling may relate to the better phonological development between 2 and 3 years of age. 
Acknowledgments. The authors thank Hanny Weersink-Braks and Margo van de Borg for their great support with the recording and transcription of the speech samples. We express our gratitude to Dr. Mieke Beers for her valuable comments on an earlier draft of this article.

\section{REFERENCES}

Beers M. The Phonology of Normally Developing and Language-Impaired Children. Amsterdam: IFOTT; 1995.

Bernhardt B, Stoel-Gammon C. Nonlinear phonology: introduction and clinical application. J Speech Hear Res. 1994;37:123-143.

Chapman KL. Phonologic processes in children with cleft palate. Cleft Palate Craniofac J. 1993;30:64-71.

Chapman KL, Hardin MA. Phonetic and phonologic skills of two-year-olds with cleft palate. Cleft Palate Craniofac J. 1992;29:435-443.

Dorf DS, Reisberg DJ, Gold HO. Early prosthetic management of cleft palate. Articulation development prosthesis: a preliminary report. J Prosthet Dent. 1985;53:222-226.

Estrem T, Broen PA. Early speech production of children with cleft palate. $J$ Speech Hear Res. 1989;32:12-23.

Fikkert P. The acquisition of Dutch phonology. In: Gillis S, De Houwer A, eds. The Acquisition of Dutch. Amsterdam: J. Benjamins Publishing Co.; 1998: 163-222.

Gnoinski WM. Infant orthopedics and later orthodontic monitoring for unilateral cleft lip and palate patients in Zurich. In: Bardach J, Morris HL, eds. Multidisciplinary Management of Cleft Lip and Palate. Philadelphia: WB Saunders; 1990:578-585.

Gruber H. Presurgical maxillary orthopedics. In: Bardach J, Morris HL, eds. Multidisciplinary Management of Cleft Lip and Palate. Philadelphia: WB Saunders; 1990:592-600.

Grunwell P. Clinical Phonology. London: Croom Helm; 1982.

Grunwell P. Principled decision making in the remediation of children with phonological disorders. In: Fletcher P, Hall D, eds. Specific Speech and Language Disorders in Children: Correlates, Characteristics and Outcomes. London: Whurr Publishers; 1993:215-240.

Grunwell P, Russell J. Phonological development in children with cleft lip and palate. Clin Linguist Phon. 1988;2:75-95.

Hotz M. Multidisziplinäre Betreuung von Patienten mit Lippen-Kiefer-GaumenSpalten in Zürich. Stomatology 1979;29:944-954.

Hotz M. Orofacial development under adverse conditions. Eur J Orthod. 1983; 5:91-103.

Hotz M, Gnoinski W, Perko M, Nussbaumer H, Hof E, Haubensak R. The Zurich approach, 1964 to 1984. In: Hotz M, Gnoinski W, Perko M, Nussbaumer H, Hof E, Haubensak R, eds. Early Treatment of Cleft Lip and Palate. Toronto: Hans Huber Publishers; 1986:42-48.
Jansonius-Schultheiss K. Twee Jaar Spraak en Taal Bij Schisis. The Hague: Holland Academic Graphics; 1999.

Konst EM, Weersink-Braks H, Rietveld T, Peters H. An intelligibility assessment of toddlers with cleft lip and palate who received and did not receive presurgical infant orthopedic treatment. J Commun Disord. 2000;33:483501.

Konst EM, Weersink-Braks H, Rietveld T, Peters H. Prelexical development of unilateral cleft lip and palate babies with reference to presurgical infant orthopaedics: a randomized prospective clinical trial. Clin Linguist Phon. 1999;13:395-407.

Kuijpers-Jagtman AM, Konst EM, Prahl C, Severens JL. A Multicentre Prospective Clinical Trial Into the Effects of Presurgical Infant Orthopaedics in Children With a Complete UCLP. Second report. Nijmegen: University Press; 1998.

Lynch JI, Fox DR, Brookshire BL. Phonological proficiency of two cleft palate toddlers with school-age follow-up. J Speech Hear Disord. 1983;48:274285.

Oller DK, Wieman LA, Doyle WJ, Ross C. Infant babbling and speech. $J$ Child Lang. 1976;3:1-11.

Russell J, Grunwell P. Speech development in children with cleft lip and palate. In: Grunwell P, ed. Analysing Cleft Palate Speech. London: Whurr Publishers; 1993:19-47.

Schlichting JEPT, van Eldik MCM, Lutje Spelberg HC, van der Meulen SJ, van der Meulen BF. Schlichting Test Voor Taalproductie: Handleiding. Lisse: Swets and Zeitlinger BV; 1995:53-54.

Severens JL, Prahl C, Kuijpers-Jagtman AM, Prahl-Andersen B. Short-term cost-effectiveness analysis of presurgical orthopedic treatment in children with complete unilateral cleft lip and palate. Cleft Palate Craniofac J. 1998; 35:222-226.

Stoel-Gammon C. Phonetic inventories, 15-24 months: a longitudinal study. $J$ Speech Hear Res. 1985;28:505-512.

Stuffins GM. Speech and mental attitudes in the older presurgical child. In: Kehrer B, Slingo T, Graf B, Bettex M, eds. Proceedings of the First International Symposium of Long Term Treatment in Cleft Lip and Palate. Bern: H. Huber Publishers; 1981:199-206.

Vihman MM, Ferguson CA, Elbert M. Phonological development from babbling to speech: common tendencies and individual differences. Appl Psycholinguist. 1986;7:3-37.

Vihman MM, Macken MA, Miller R, Simmons H, Miller J. From babbling to speech: a reassessment of the continuity issue. Language. 1985;61:395-443.

Vihman MM, Miller R. Words and babble at the threshold of language acquisition. In: Smith MD, Locke JL, eds. The Emergent Lexicon. The Child's Development of a Linguistic Vocabulary. San Diego: Academic Press; 1988: 151-183.

Winters JC, Hurwitz DJ. Presurgical orthopedics in the surgical management of unilateral cleft lip and palate. Plast Reconstr Surg. 1995;95:755-764.

Witzel MA, Salyer KE, Ross RB. Delayed hard palate closure: the philosophy revisited. Cleft Palate J. 1984;21:263-269. 